\title{
Effectiveness and Safety of Posterior Vaginal Repair with Single- Incision, Ultralightweight, Monofilament Propylene Mesh: First Evidence from a Case Series with Short-Term Results
}

\author{
Francesco Deltetto, ${ }^{1}$ Alessandro Favilli $\mathbb{D}^{2},{ }^{2}$ Giovanni Buzzaccarini $\left(\mathbb{D},{ }^{3}\right.$ \\ and Amerigo Vitagliano $\mathbb{1}^{4}$ \\ ${ }^{1}$ San Luca Nursing Home, Torino, Italy \\ ${ }^{2}$ Department of Obstetrics and Gynecology, AOUI Verona, Verona, Italy \\ ${ }^{3}$ Gynecological Division, San Camillo Hospital, Trento, Italy \\ ${ }^{4}$ Department of Women and Children's Health, Unit of Gynecology and Obstetrics, University of Padua, 35128 Padua, Italy \\ Correspondence should be addressed to Amerigo Vitagliano; amerigovitagliano.md@gmail.com
}

Received 23 April 2020; Revised 11 November 2020; Accepted 18 December 2020; Published 4 January 2021

Academic Editor: Gail Mahady

Copyright (c) 2021 Francesco Deltetto et al. This is an open access article distributed under the Creative Commons Attribution License, which permits unrestricted use, distribution, and reproduction in any medium, provided the original work is properly cited.

\begin{abstract}
Objective. The use of transvaginal mesh is controversial, and over time, multiple surgical methods for the treatment of posterior vaginal prolapse (PVP) have been proposed including different surgical approaches and techniques. To date, no clear conclusion has been reached about the use of mesh for reinforcing transvaginal posterior repair. The aim of this study was to evaluate the feasibility, safety, and effectiveness of a novel, ultralightweight mesh for the treatment of PVP. Methods. We performed a single-center, prospective observational study on consecutive patients referred for primary or recurrent, symptomatic stage II PVP (according to the international Pelvic Organ Prolapse Quantification System) from April 2017 to September 2018. In all patients, transvaginal posterior repair was augmented with a single-incision, isoelastic polypropylene mesh. Data about the postoperative outcomes were collected until December 2019. Results. A total number of 15 patients were included. The median follow-up after surgery was 18 months $(\mathrm{IQR}=14)$. Surgery was completed in all cases without complications. Regarding the anatomical outcomes (as measured according to POP-q classification), a significant improvement was observed in terms of $\mathrm{Bp}, \mathrm{D}$, and $\mathrm{C}(p<0.05)$. The functional outcomes were significantly ameliorated after surgery, with a reduction of bulge symptom, stypsis, incomplete evacuation, and excessive staining $(p<0.05)$. The quality of life was significantly improved in the majority of patients $(p<0.05)$. Median patients' satisfaction rate was $100 \%(I Q R=22.5 \%)$. Neither early nor late postoperative complications occurred. Conclusions. Single-incision, ultralightweight polypropylene meshes were safe and highly effective in the treatment of PVP. As our study has some limitations, further large, controlled studies are needed.
\end{abstract}

\section{Introduction}

Pelvic organ prolapse (POP) refers to the descent or herniation of one or more pelvic organs from their normal attachment sites or their normal position in the pelvis [1]. POP may variably involve the anterior vaginal wall (cystocele), the posterior vaginal wall (rectocele, enterocele), the uterus (uterine prolapse), and the vaginal apex (apical vaginal prolapse) $[1,2]$.
POP is a common condition, affecting up to $50 \%$ of parous women and being associated with high healthcare costs [3]. At present, the lifetime risk for women undergoing POP surgery is estimated between $6.3 \%$ and $19 \%$ and is expected to further increase in the near future $[4,5]$. For these reasons, the development of effective strategies for treating POP has gained high social priority.

The second most common type of POP is posterior vaginal prolapse (PVP), whose incidence is estimated in 5.7 new 
cases per 100 women-year $[6,7]$. When symptomatic, PVP can be associated with rectal pain and defecatory dysfunctions (such as constipation, tenesmus, splinting, and fecal incontinence), potentially causing a serious deterioration of patients' quality of life $[8,9]$.

The use of transvaginal mesh is controversial, and over time, multiple surgical methods have been proposed for PVP including different surgical approaches (i.e., transvaginal, transperineal, and transanal) and techniques (i.e., native tissue repair, augmentation with biological graft, or synthetic mesh) $[10,11]$. Recent studies were in agreement in demonstrating the superiority of transvaginal repair compared to other surgical approaches for PVP, as the transvaginal approach was associated with the lowest risk of prolapse recurrence [10]. Nevertheless, no clear conclusion has been reached about the use of mesh for reinforcing transvaginal posterior repair (TVP). On the one hand, transvaginal mesh surgery (TVM) may reduce the risk of prolapse recurrence compared to native tissue repair. On the other hand, TVM may be associated with a higher risk of surgical complications including mesh extrusion, perineal pain, and dyspareunia [10, 12]. Therefore, the benefit/risk ratio for TVM is the fundamental point which is currently under scientific scrutiny.

Current evidence on the effectiveness and harms of TVM is mainly limited by the problem that previous studies employed outdated meshes with high weight or transobturator harms, most of whom have been already withdrawn from the market. In recent times, more technological ultralightweight mesh kits have been developed for treating PVP, with some potential advantages compared to the older ones. First, the lighter weight of the meshes may reduce the risk of vaginal extrusion. Moreover, these meshes can be positioned using a minimally invasive technique with a single surgical incision, potentially minimising the surgical trauma and improving patients' postoperative outcomes $[13,14]$.

Here, we present the first series of patients who undergone TVP with a novel, ultralightweight mesh kit with a median follow-up of 18 months.

\section{Materials and Methods}

2.1. Study Design. The present was a single-center case series realized at Gynecological Division, San Camillo Hospital, Trento, Italy. We enrolled consecutive patients referred for PVP in whom TVM was indicated from April 2017 to September 2018. All participants gave written informed consent to use their data for research purposes. The study was exempted from institutional review board (IRB) approval because its design was observational (i.e., without any modification of the routine clinical practice) and all data was anonymized before analysis. All the surgical procedures were performed by a single skilled surgeon (FD) with more than 500 previous TVM surgery procedures. The study was conducted following the IUGA/International Continence Society (ICS) joint report on the terminology for reporting outcomes of surgical procedures for pelvic organ prolapse [15]. Cost analysis was not performed.
2.2. Participants. We included patients with a diagnosis of primary or recurrent, symptomatic stage II PVP according to the international Pelvic Organ Prolapse Quantification System (POP-Q) [16]. All patients with rectocele and/or enterocele were eligible. Patients were included exclusively if able to give written informed consent. Exclusion criteria were as follows: malignant diseases, previous TVM surgery, neuromuscular diseases, dementia, and history of chronic pelvic pain.

2.3. Mesh Material. In all patients, transvaginal posterior repair was augmented with an isoelastic mesh (InGYNious, A.M.I., Feldkirch, Austria). It is ultralightweight, monofilament polypropylene mesh $\left(21 \mathrm{~g} / \mathrm{m}^{2}\right)$ with a hexagonal structure and six-point suture fixation (three-level support), consisting of extralarge micropores of 100 to $150 \mu \mathrm{m}$ and macropores with diameters of 1.9 (uniform) to $2.8 \mathrm{~mm}$ (maximum) (Figure 1).

2.4. Surgical Technique. After infiltration with adrenaline solution into the subfascial posterior vaginal wall, a vertical incision is performed with a cold blade scalpel. The vaginal edges are grabbed with Kocher forceps leaving the fascia adherent to the mucosa.

Now, a delicate digital detachment is performed laterally and downwards until the fibrous nucleus of the perineum. Upwards, lateral detachment slides behind the cervix and the Douglas peritoneum. In the case of conspicuous enterocele, a peritoneum opening may occur and is sutured with a continuous absorbable suture.

The lateral detachment reaches the yellow perirectal fat tissue. Then, the index finger is pushed downwards to the ischial spine. Using the Mayo scissors and the index finger, the bone spike is reached. At this point, the preparation of the sacrospinous ligament is performed by pressing the finger on it. The rectum is then medialized. Slipping down the sacrospinous ligament, the surface of the iliococcygeus muscle is easily identified for the reconstitution of the II level of De Lancey.

At this point, laterally to the fibrous nucleus of the perineum, the deep perineal transverse muscles are found for the reconstruction of the III level.

With the i-Stitch device (A.M.I., Feldkirch, Austria; Figure 2), the sacrospinous ligament and the ileococcygeal muscle are reached to put polypropylene sutures and these sutures pass through the mesh too. One end of the sutures fixed to the ligament sacrospinous goes through the cervix. Then, the distal mesh is connected to the deep transverse perineum muscle. Now, the sutures are tied. All the steps are bilateral. At the end, the vaginal suture is performed.

Repairing a posterior defect after hysterectomy and then without the cervix, the mesh does not have to hook to the vagina in order to reduce extrusion risk. Another absorbable suture is passed through the sacrospinous ligaments to fix up and suspend the vagina. They are tied after the vaginal closure as the final step of the intervention.

2.5. Data Collection and Follow-Up. Two authors with adequate training in pelvic surgery (F.D. and A.V.) completed 


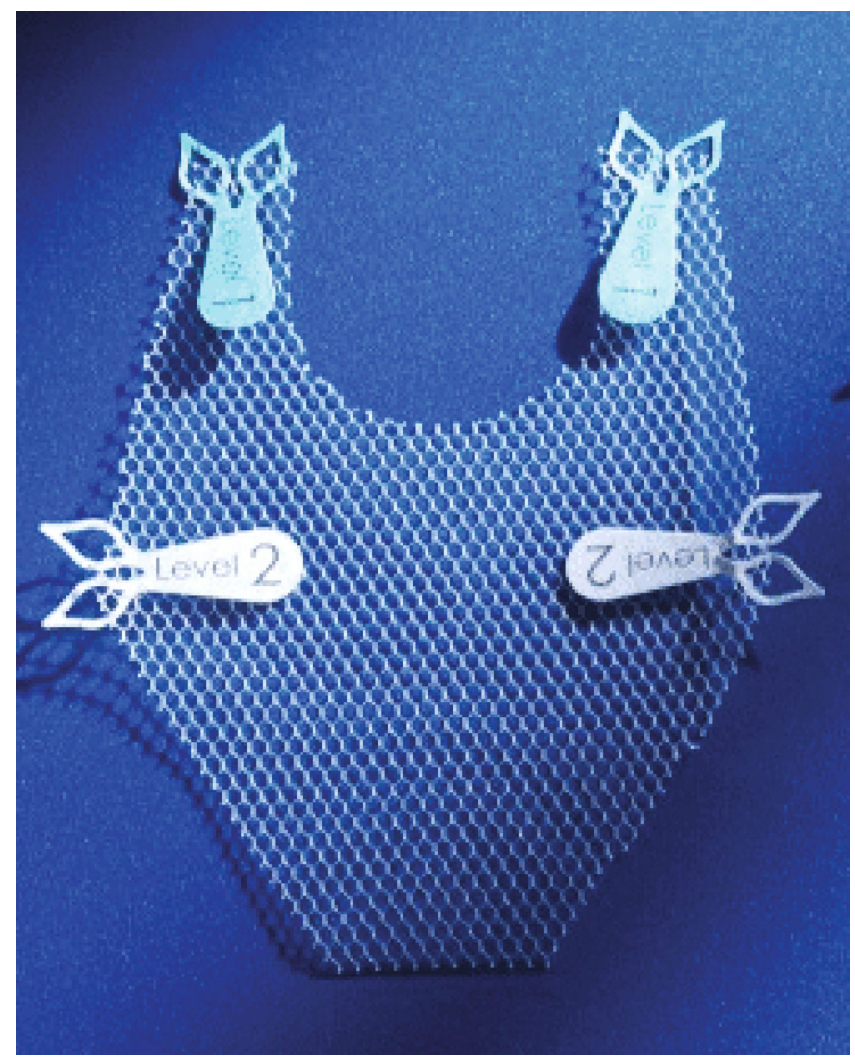

Figure 1: InGYNious (A.M.I., Feldkirch, Austria) ultralightweight, monofilament polypropylene mesh $\left(21 \mathrm{~g} / \mathrm{m}^{2}\right)$, with a hexagonal structure and six-point suture fixation (three-level support), consisting of extralarge micropores of 100 to $150 \mu \mathrm{m}$ and macropores with diameters of 1.9 (uniform) to $2.8 \mathrm{~mm}$ (maximum).

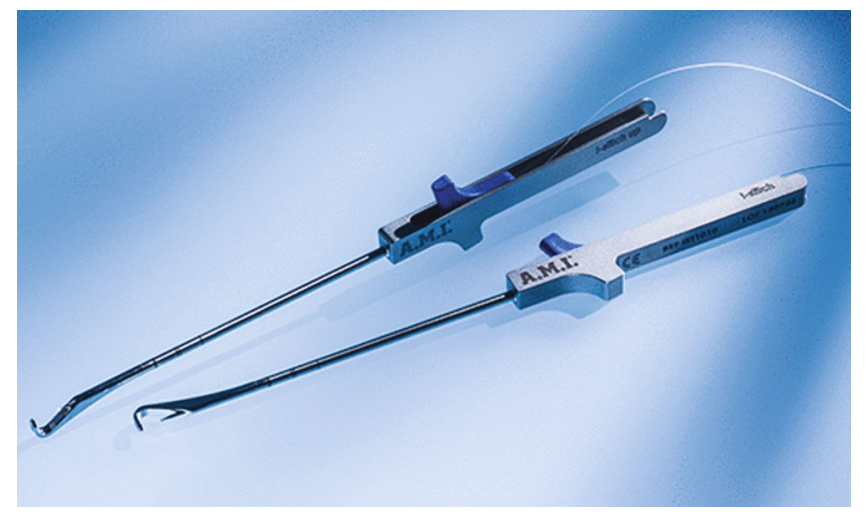

FIGURE 2: I-stitch. Reusable instrument for fixation of suture material to the tissue.

the data collection prospectively. Data included patients' general features (i.e., age, BMI, and menopausal status), indication to surgery (including the type of POP, POPrelated symptoms, quality of life, and sexual activity), perioperative data (i.e., duration of the intervention, blood loss, perioperative complications, length of hospitalization, and need for analgesia), and postoperative data (objective anatomical outcomes, functional outcomes, sexual life after surgery, degree of satisfaction about the VPM, quality of life, and postoperative complications). The sexual function after surgery was assessed by using a validated questionnaire (Pelvic Organ Prolapse/Urinary Incontinence Sexual Questionnaire (PISQ-12)) [17].

The evaluation timeline was very early ( 1 and 3 months after surgery), early (every 3 months until the completion of the first year after surgery), and intermediate (every 3 months after the first year after surgery). Information was collected clinically (by gynecological check-up) and by periodic telephonic 
IUGA flow diagram

\section{Enrollment}

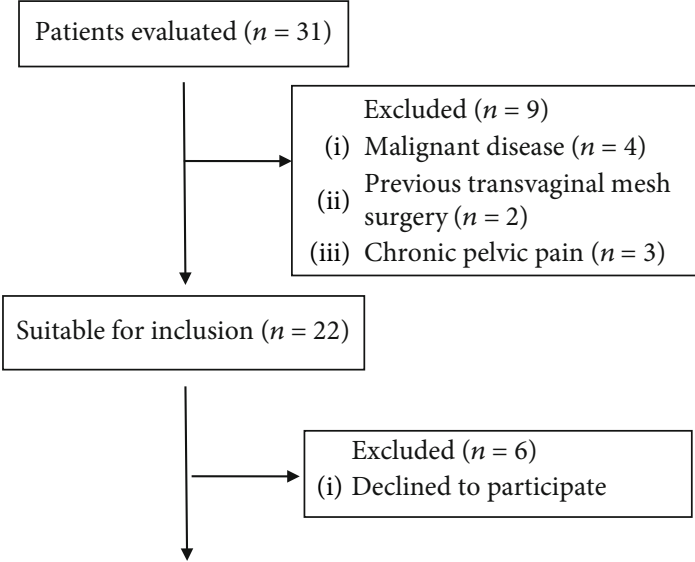

Agreed to participate $(n=16)$

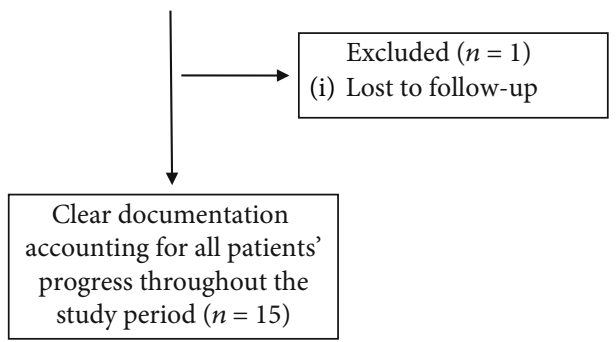

Figure 3: IUGA flow-diagram of patients' enrollment.

interviews (every 6 months) until December 2019. All the data were inserted in an electronic database in Excel format.

2.6. Statistical Analysis. Statistical analysis was performed using the Statistical Package for Social Sciences (SPSS) v. 22.0 (IBM Inc., Armonk, NY, USA). Continuous variables were reported as median with interquartile range (IQR). Qualitative variables were presented as frequencies (absolute) and percentages (\%). Differences between preoperative and postoperative evaluations were analyzed by using the Wilcoxon signed-rank test for matched pairs for numerical variables and the $\mathrm{McNemar}$ test for categorical variables. The level of significance was set at $p=0.05$. Power analysis was not feasible.

\section{Results}

We assessed for eligibility a total number of 31 patients. 15 patients were finally included after applying our inclusion and exclusion criteria (Figure 3 ). The median age at the time of surgery was 69 years (IQR = 14), and the median BMI was $25(\mathrm{IQR}=7)$. Nine patients had a history of pelvic prolapse surgery ( $n=4$ vaginal hysterectomy, $n=3$ vaginal hysterectomy plus posterior repair, and $n=2$ cystocele repair). The indication for TVM was symptomatic enterorectocele in 10 cases $(66.7 \%)$, rectocele in four cases $(36.3 \%)$, and isolated enterocele in a single patient $(6.7 \%)$. The most common symptoms were bulge $(n=15,100 \%)$, incomplete evacuation
( $n=11,73.3 \%)$, stypsis $(n=10,66.7 \%)$, excessive staining $(n=10,66.7 \%)$, and urgency $(n=10,66.7 \%)$ (Table 1$)$.

The median duration of the surgical procedure was 50 minutes $(\mathrm{IQR}=20)$, and the median blood loss was $20 \mathrm{cc}$ $(\mathrm{IQR}=10)$. The median hospital stay was 2 days $(\mathrm{IQR}=1)$, and the median time to return to daily activities was 18 days $(\mathrm{IQR}=8)$. The median follow-up after surgery was 18 months (IQR = 14). In a single case, the follow-up was interrupted before the end of the study (i.e., 15 months after surgery) due to cerebral hemorrhage and sudden death. No perioperative complications were recorded.

Regarding the anatomical outcomes (as measured according to POP-q classification at the last follow-up visit compared to preoperative evaluation), a significant improvement was observed in terms of $\mathrm{Bp}(-2(\mathrm{IQR}=1)$ vs. 2 $(\mathrm{IQR}=1), p=0.001), \operatorname{Ap}(-2(\mathrm{IQR}=1)$ vs. $2(\mathrm{IQR}=2), p=$ $0.001), \mathrm{D}(-3(\mathrm{IQR}=4)$ vs. $2(\mathrm{IQR}=3), p=0.003), \mathrm{C}(-4$ $(\mathrm{IQR}=1)$ vs. $0(\mathrm{IQR}=6), p=0.005)$ with no differences in $\mathrm{Ba}(-2(\mathrm{IQR}=1)$ vs. $-2(\mathrm{IQR}=1), p=\mathrm{ns})$, and $\mathrm{Aa}(-2$ $(\mathrm{IQR}=1)$ vs. $-2(\mathrm{IQR}=1), p=\mathrm{ns})$. Additionally, the QoL was significantly improved in the majority of patients (i.e., ameliorated in nine patients, unchanged in three patients, worsened in one patient, and not estimable in a single case $(p=0.03))$.

The functional outcomes were considerably ameliorated after surgery, with a significant reduction of bulge symptom ( $20 \%$ vs. $100 \%, p=0.001)$, stypsis ( $13 \%$ vs. $66.7 \%, p=0.01)$, incomplete evacuation ( $13 \%$ vs. $73.3 \%, p=0.007)$, and 
TABle 1: Preoperative data.

\begin{tabular}{lccccccccccccc}
\hline Patient & $\begin{array}{c}\text { Age at } \\
\text { surgery }\end{array}$ & Parity & $\begin{array}{c}\text { BMI at } \\
\text { surgery }\end{array}$ & $\begin{array}{c}\text { Menopause } \\
\text { status }\end{array}$ & $\begin{array}{c}\text { HRT } \\
\text { usage }\end{array}$ & Smoking & $\begin{array}{c}\text { Chronic } \\
\text { cough }\end{array}$ & $\begin{array}{c}\text { Previous } \\
\text { Gyn } \\
\text { surgery }\end{array}$ & $\begin{array}{c}\text { Defects } \\
\text { Anatomic } \\
\text { entity }\end{array}$ & $\begin{array}{c}\text { Functional } \\
\text { entity }\end{array}$ & $\begin{array}{c}\text { Sexual } \\
\text { activity }\end{array}$ \\
QoL
\end{tabular}

Legend: HRT: hormone replacement therapy. Previous Gyn surgery: H: hysterectomy; P: prolapse correction; C: cystocele correction. Defects: E: enterocele; R: rectocele; A: anterior wall prolapse. Anatomic entity: the number is representative of POP-Q anatomic classification (Pelvic Organ Prolapse Quantification) following the International Continence Society (ICS). The numbers are in centimeter, in order: Aa (anterior vaginal wall caudally), Ba (anterior vaginal wall cranially), C (more declive vaginal point), D (posterior fornix), Ap (posterior vaginal wall caudally), and Bp (posterior vaginal wall cranially). Positive numbers mean a more prolapsed structure; negative numbers mean a more cranially structure. Functional entity: S: stypsis; D: digital use; E: incomplete evacuation; P: excessive straining (all considered for symptoms more frequently than $1 /$ month); B: vaginal bulge (b for few symptoms); U: urgency. QoL: quality of life scale; ++/: excellent; +/: food; +/-: tolerated; /-: poor; /-: terrible.

\begin{tabular}{|c|c|c|c|c|c|c|c|c|c|c|c|c|c|}
\hline Patient & $\begin{array}{c}\text { Duration } \\
\text { of } \\
\text { operation (min) }\end{array}$ & $\begin{array}{c}\text { Blood } \\
\text { loss } \\
(\mathrm{mL})\end{array}$ & $\begin{array}{c}\text { Hospital } \\
\text { stay } \\
\text { (days) }\end{array}$ & $\begin{array}{c}\text { Return } \\
\text { to daily } \\
\text { activities (days) }\end{array}$ & $\begin{array}{l}\text { Peri-OP } \\
\text { COMPL }\end{array}$ & $\begin{array}{c}\text { Anatomic } \\
\text { entity after } \\
\text { surgery }\end{array}$ & $\begin{array}{c}\text { Functional } \\
\text { entity after } \\
\text { surgery }\end{array}$ & $\begin{array}{c}\text { Sexual } \\
\text { activity } \\
\text { after surgery }\end{array}$ & $\begin{array}{c}\text { QoL } \\
\text { after } \\
\text { surgery }\end{array}$ & $\begin{array}{l}\text { Post- } \\
\text { operative } \\
\text { pain }\end{array}$ & $\begin{array}{c}\text { Satisfaction } \\
\quad(0-100)\end{array}$ & $\begin{array}{l}\text { Post-OP } \\
\text { COMPL }\end{array}$ & $\begin{array}{c}\text { Follow-up } \\
\text { length } \\
\text { (months) }\end{array}$ \\
\hline 1 & 35 & 20 & 3 & 20 & None & $0-2-2-3-1-2$ & - & $=$ & ++1 & No pain & 100 & None & 32 \\
\hline 2 & 45 & 30 & 2 & 14 & None & $00-3-3-1-2$ & $\mathrm{U}$ & $=$ & +1 & No pain & 100 & None & 32 \\
\hline 3 & 45 & 20 & 2 & 34 & None & $-2-20100$ & - & $=$ & $1-$ & $\begin{array}{l}\text { Provoked } \\
\text { pain only } \\
\text { Pain }\end{array}$ & 40 & None & 30 \\
\hline 4 & 55 & 30 & 2 & 29 & None & $-2-4-5020$ & BSEP & + & 1- & $\begin{array}{c}\text { during } \\
\text { physical activities }\end{array}$ & 30 & None & 28 \\
\hline 5 & 40 & 20 & 2 & 18 & None & $00-4-4-1-2$ & B & + & +1 & $\begin{array}{c}\text { physical activities } \\
\text { No pain }\end{array}$ & 90 & None & 21 \\
\hline 6 & 40 & 20 & 2 & 23 & None & $-1-1-4-4-1-3$ & BSEPU & No & $1-$ & $\begin{array}{l}\text { Provoked } \\
\text { pain only }\end{array}$ & 70 & None & 20 \\
\hline 7 & 60 & 30 & 4 & 36 & None & $0-1-3-40-2$ & U & No & +1 & No pain & 100 & None & 17 \\
\hline 8 & 60 & 200 & 2 & 16 & None & $-2-1-4 /-2-3$ & - & No & +1 & No pain & 100 & None & 16 \\
\hline 9 & 50 & 20 & 2 & 21 & None & $-1-2-3-3-2-3$ & - & No & ++1 & No pain & 90 & None & 16 \\
\hline 10 & 35 & 20 & 2 & 15 & None & $-2-3-3-3-3-3$ & - & $=$ & ++1 & No pain & 100 & None & 16 \\
\hline 11 & 65 & 30 & 2 & 16 & None & $-1-2-3-3-2-2$ & $\mathrm{U}$ & + & ++1 & No pain & 100 & None & 15 \\
\hline 12 & 50 & 20 & 2 & 14 & None & $-1-1-4-4-2-2$ & - & No & +1 & No pain & 100 & None & 15 \\
\hline 13 & 60 & 30 & 3 & 19 & None & $-1-2-3-3-1-2$ & $\mathrm{U}$ & No & +1 & No pain & 80 & None & 31 \\
\hline 14 & 40 & 30 & 3 & 15 & None & $-2-2-4-4-2-3$ & - & No & ++1 & No pain & 100 & None & 16 \\
\hline 15 & 55 & 20 & 2 & 12 & None & $-1-1-4-4-2-2$ & None & $\begin{array}{c}\text { Not } \\
\text { Known }\end{array}$ & $\begin{array}{c}\text { Not } \\
\text { Known }\end{array}$ & No pain & 100 & None & 15 \\
\hline
\end{tabular}

Figure 4: Perioperative data. Peri-OP COMPL: perioperative complications. Anatomic entity: the number are representative of POP-Q anatomic classification (Pelvic Organ Prolapse Quantification) following the International Continence Society (ICS). The numbers are in centimeter, in order: Aa (anterior vaginal wall caudally), Ba (anterior vaginal wall cranially), C (more declive vaginal point), D (posterior fornix), Ap (posterior vaginal wall caudally), and $\mathrm{Bp}$ (posterior vaginal wall cranially). Positive numbers mean a more prolapsed structure; negative numbers mean a more cranially structure. In red, we highlighted the improvement in anatomic defects; in blue, the worsening. Functional entity: S: stypsis; D: digital use; E: incomplete evacuation; P: excessive straining (all considered for symptoms more frequently than 1/month); B: vaginal bulge (b for few symptoms); U: urgency. As above, in blue, the worsening of symptoms. QoL: quality of life scale; ++/: excellent; +/: good; +/-: tolerated; /-: poor; /-: terrible. In red, we highlighted the improvement in quality of life score; in blue, the worsening. Post-OP COMPL: postoperative complications. 
excessive staining ( $13 \%$ vs. $66.7 \%, p=0.01)$. Differently, a moderate but nonstatistically significant amelioration in the urge symptom (33.3\% vs. $66.7 \%, p=n s)$ and digital evacuation ( $0 \%$ vs. $13.3 \%)$ was recorded.

Only seven out of 15 reported an active sexual life before surgery, of whom three experienced an improvement in sexual activity and four did not report any change after surgery ( $p=n s$ ). In the remaining patients, the sexual activity before and after surgery was not measurable. No patient experienced postoperative dyspareunia.

Importantly, the majority of patients were considerably satisfied with their postoperative outcomes, with a median satisfaction rate of $100 \%(\mathrm{IQR}=22.5 \%)$. Finally, neither early nor late postoperative complications occurred. All data about perioperative and postoperative outcomes are reported in Figure 4.

\section{Discussion}

4.1. Main Findings. To the best of our knowledge, this is the first study investigating the feasibility, safety, and effectiveness of an ultralightweight mesh (InGYNious) for PVP repair. Importantly, the median duration of surgery was 50 minutes and did not exceed 65 minutes in any case, suggesting a fast and reproducible surgical technique. In particular, when comparing our data with those from other studies on laparoscopic PVP mesh repair, the time needed for surgery was considerably lower in our experience (mean time of laparoscopic POP repair $>100$ minutes) $[10,18]$. Additionally, the use of spinal anaesthesia and no requirement of Trendelenburg position represent considerable advantages of our technique for surgery in elderly women.

Moreover, our experience substantiates the safety of this novel surgical approach, as the procedures were completed with minimal blood loss $($ median $=20 \mathrm{cc}$ ) and no perioperative complications. These findings are inconsistent with those from previous studies on vaginal PVP repair, in whom mesh augmentation was associated with a high risk of surgical complications $[10,18-21]$. In this respect, we may speculate that our positive results are due to the use of a single-incision, simplified technique, with minimal surgical invasivity and a lower risk of complications.

Notably, we found a significant improvement in both anatomical (i.e., Bp, Ap, D, and C as measured with POP-q classification) and functional aspects after surgery (i.e., bulge symptom, stypsis, incomplete evacuation, and excessive staining), resulting in improved QoL and high patients' satisfaction rate. We believe these two latter points to be of crucial importance, as the primary aim of POP surgery is the improvement of the physical, psychological, and social wellbeing of patients, rather than the mere resolution of anatomic defects.

Additionally, after a median follow-up of 18 months, we did not record any mesh-related surgical complication, including not even a single case of vaginal extrusion. These data may reassure both physicians and patients about the safety of vaginal augmentation with novel, macroporous, ultralightweight polypropylene meshes. For the sake of clarity, we need to stress that current evidence on the safety of
TVM is mainly based on trials that are not exempted from limitations. The main issue is the high variability within studies in terms of mesh characteristics and surgical techniques for vaginal mesh augmentation [22]. Such a methodological heterogeneity may inevitably expose to variability in the intervention effects and, therefore, to bias in pooled effect estimates. For instance, we may speculate that ultraweight macroporous meshes, as positioned under the pubocervical fascia, may reduce the risk of vaginal extrusion. Nevertheless, our hypotheses need future confirmation by well-conducted trials with homogeneous interventions.

4.2. Strengths and Limitations. The key strengths of this study are the long follow-up, the use of a single surgical technique (including a single mesh type), and the involvement of a single surgeon with high expertise in POP surgery. The main weaknesses are inherent to study design (i.e., lack of a control group) and small sample size, potentially limiting drawing firm conclusions from the data.

\section{Conclusions}

Single-incision, ultralightweight polypropylene meshes were safe and highly effective in the treatment of PVP. TVM was associated with significant improvement in the anatomical and functional outcomes, better QoL, and high patients' satisfaction rate. Moreover, the surgical time was limited and no perioperative and postoperative complications occurred. Accordingly, TVM with ultralightweight polypropylene meshes may be considered as a promising option for PVP repair. As our study has some limitations, further large, controlled studies with long-term follow-up are needed to confirm our findings.

\section{Data Availability}

We declare that all data are available within the study tables.

\section{Conflicts of Interest}

All authors have no conflicts of interest to disclose.

\section{References}

[1] A. M. Weber and H. E. Richter, "Pelvic organ prolapse," Obstetrics and Gynecology, vol. 106, no. 3, pp. 615-634, 2005.

[2] I. Kuncharapu, B. A. Majeroni, and D. W. Johnson, "Pelvic organ prolapse," American Family Physician, vol. 81, no. 9, pp. 1111-1117, 2010.

[3] S. Hagen, D. Stark, C. Maher, and E. Adams, "Conservative management of pelvic organ prolapse in women," Cochrane Database of Systematic Reviews, no. 4, article CD003882, 2006.

[4] E. Løwenstein, B. Ottesen, and H. Gimbel, "Incidence and lifetime risk of pelvic organ prolapse surgery in Denmark from 1977 to 2009," International Urogynecology Journal, vol. 26, no. 1, pp. 49-55, 2015.

[5] F. J. Smith, D. A. Holman, R. Moorin, and N. Tsokos, "Lifetime risk of undergoing surgery for pelvic organ prolapse," Obstetrics \& Gynecology, vol. 116, no. 5, pp. 1096-1100, 2010. 
[6] J. Luo, K. A. Larson, D. E. Fenner, J. A. Ashton-Miller, and J. O. DeLancey, "Posterior vaginal prolapse shape and position changes at maximal Valsalva seen in 3-D MRI-based models," International Urogynecology Journal, vol. 23, no. 9, pp. 13011306, 2012.

[7] D. Altman, A. López, J. Kierkegaard et al., “Assessment of posterior vaginal wall prolapse: comparison of physical findings to cystodefecoperitoneography," International Urogynecology Journal, vol. 16, no. 2, pp. 96-103, 2005.

[8] C. L. Grimes and E. S. Lukacz, "Posterior vaginal compartment prolapse and defecatory dysfunction: are they related?," International Urogynecology Journal, vol. 23, no. 5, pp. 537-551, 2012.

[9] G. A. Digesu, C. Chaliha, S. Salvatore, A. Hutchings, and V. Khullar, "The relationship of vaginal prolapse severity to symptoms and quality of life," BJOG : An International Journal of Obstetrics and Gynaecology, vol. 112, no. 7, pp. 971-976, 2005.

[10] A. Mowat, D. Maher, K. Baessler, C. Christmann-Schmid, N. Haya, and C. Maher, "Surgery for women with posterior compartment prolapse," Cochrane Database of Systematic Reviews, vol. 3, no. 3, article CD012975, 2018.

[11] K. J. Ko and K. S. Lee, "Current surgical management of pelvic organ prolapse: strategies for the improvement of surgical outcomes," Investigative and Clinical Urology, vol. 60, no. 6, pp. 413-424, 2019.

[12] C. Glazener, S. Breeman, A. Elders et al., "Clinical effectiveness and cost-effectiveness of surgical options for the management of anterior and/or posterior vaginal wall prolapse: two randomised controlled trials within a comprehensive cohort study results from the PROSPECT Study," Health Technology Assessment, vol. 20, no. 95, pp. 1-452, 2016.

[13] A. Brandt, A. Kuszka, A. Niesel et al., "1-year outcome after treatment of uterovaginal prolapse with a 6-point fixation mesh," Neurourology and Urodynamics, vol. 38, no. 4, pp. 1129-1134, 2019.

[14] P. Ferry, P. Bertherat, A. Gauthier et al., "Transvaginal treatment of anterior and apical genital prolapses using an ultra lightweight mesh: Restorelle $\left.{ }^{\circledR}\right)$ Direct Fix ${ }^{\mathrm{TM}}$. A retrospective study on feasibility and morbidity," Journal of Gynecology Obstetrics and Human Reproduction, vol. 47, no. 9, pp. 443449, 2018.

[15] P. Toozs-Hobson, R. Freeman, M. Barber et al., “An International Urogynecological Association (IUGA)/International ontinence ociety (ICS) joint report on the terminology for reporting outcomes of surgical procedures for pelvic organ prolapse," International Urogynecology Journal, vol. 23, no. 5, pp. 527-535, 2012.

[16] C. Persu, C. R. Chapple, V. Cauni, S. Gutue, and P. Geavlete, "Pelvic Organ Prolapse Quantification System (POP-Q) - a new era in pelvic prolapse staging," Journal of Medicine and Life, vol. 4, no. 1, pp. 75-81, 2011.

[17] R. G. Rogers, K. W. Coates, D. Kammerer-Doak, S. Khalsa, and C. Qualls, "A short form of the Pelvic Organ Prolapse/Urinary Incontinence Sexual Questionnaire (PISQ-12)," International Urogynecology Journal and Pelvic Floor Dysfunction, vol. 14, no. 3, pp. 164-168, 2003.

[18] C. M. Glazener, S. Breeman, A. Elders et al., "Mesh, graft, or standard repair for women having primary transvaginal anterior or posterior compartment prolapse surgery: two parallel-group, multicentre, randomised, controlled trials
(PROSPECT)," The Lancet, vol. 389, no. 10067, pp. 381392, 2017.

[19] N. D. Bedford, E. I. Seman, R. T. O'Shea, and M. J. Keirse, "Long-term outcomes of laparoscopic repair of cystocoele," The Australian and New Zealand Journal of Obstetrics of Gynaecology, vol. 55, no. 6, pp. 588-592, 2015.

[20] J. P. Lucot, M. Cosson, G. Bader et al., "Safety of vaginal mesh surgery versus laparoscopic mesh sacropexy for cystocele repair: results of the prosthetic pelvic floor repair randomized controlled trial," European Urology, vol. 74, no. 2, pp. 167-176, 2018.

[21] M. Bologna, A. Vitagliano, and M. Cervigni, "Re: Jean-Philippe Lucot, Michel Cosson, Georges Bader, et al. Safety of vaginal mesh surgery versus laparoscopic mesh sacropexy for cystocele repair: results of the prosthetic pelvic floor repair randomized controlled trial. Eur Urol 2018;74:167-76: Is There Enough Evidence To Prove Higher Safety of Laparoscopic Sacropexy in Comparison to Vaginal Surgery for Cystocele Mesh Repair?," European Urology, vol. 74, no. 3, pp. e71-e72, 2018.

[22] P. Dällenbach, "To mesh or not to mesh: a review of pelvic organ reconstructive surgery," International Journal of Women's Health, vol. 7, pp. 331-343, 2015. 